\title{
Basic Theoretical Methods in Microwave Plasma Polarimetry: Quasi-Isotropic Approximation, Stokes Vector Formalism and Complex Polarization Angle Method
}

\author{
Yu.A. Kravtsov ${ }^{1,2)}$, B. Bieg ${ }^{2)}$, K.Yu.Bliokh ${ }^{3,4)}$ and M.Hirsch ${ }^{5)}$ \\ 1) Space Research Institute, Profsoyuznaya St. 82/34, Moscow 117997, Russia, \\ 2) Institute of Physics, Maritime University of Szczecin, Waly Chrobrego 1/.,70-500 Szczecin, Poland, \\ 3) Institute of Radio Astronomy, 4 Krasnoznamyonnaya St., Kharkov 61002, Ukraine, \\ 4) Optical Engineering Laboratory, Faculty of Mechanical Engineering, \\ Technion-Israel Institute of Technology, Haifa 32000, Israel \\ 5) Max Planck Institute for Plasma Physics, Greifswald, Wendelsteinstrasse D-17491, Germany.
}

\begin{abstract}
Three different theoretical approaches are presented: quasi-isotropic approximation (QIA), Stokes vector formalism and complex polarization angle method, which allow describing polarization of electromagnetic waves in weakly anisotropic plasma. QIA stems directly from the Maxwell equations under assumption of weak anisotropy and has a form of coupled differential equations for the transverse components of the electromagnetic wave field. Being applied to high frequency (microwave or IR) electromagnetic waves in magnetized plasma, QIA describes combined action of Faraday and Cotton-Mouton phenomena. QIA takes into account curvature and torsion of the ray, describes normal modes conversion in the inhomogeneous plasma and allows specifying area of applicability of the method. In distinction to QIA, Stokes vector formalism (SVF) deals with quantities, quadratic in a wave field. It is shown (and this is the main result of the paper) that equation for Stokes vector evolution can be derived directly from QIA. This evidences deep unity of two seemingly different approaches. In fact QIA suggests somewhat more information than SVF; in particular, it describes the phases of both transverse components of the electromagnetic field, whereas SVF operates only with the phase difference.

At last, the coupled equations of the quasi-isotropic approximation can be reduced to a single equation for complex polarization angle (CPA), which describes both the shape and orientation of the polarization ellipse. In turn, equation for CPA allows obtaining equations for traditional parameters of polarization ellipse, which in fact are equivalent to the equation for Stokes vector evolution. It is pointed out that every method under discussion has its own advantages plasma polarimetry.
\end{abstract}

Keywords: Plasma; polarimetry; Stokes vector; quasi-isotropic approximation.

PACS: $41.20 . \mathrm{Jb}$ 42.25.Ja 52.70.-m 52.70.Ds 52.70.Gw 52.70.Kz

\section{INTRODUCTION}

Interaction of electromagnetic normal modes in inhomogeneous plasma and their polarization state evolution was studied by Budden [1,2], who suggested the system of coupled wave equations for the components of the electromagnetic wave field. Coupled wave equations for weakly anisotropic media, were specified in [3] in the form of quasi-isotropic approximation (QIA) of the geometrical optics method. QIA was developed in depth in subsequent publications [4,5] and briefly outlined in the books [6,7].

An alternative approach - "Stokes vector formalism" (SVF) - was initiated in the papers [8,9]. This approach, operating with the Stokes vector, was applied for analysis of electromagnetic waves polarization in fibers [10] and in the inhomogeneous plasma [11-14]. Comparative analysis of two approaches mentioned have been performed by Serge [15], who has analyzed advantages and shortcomings of each technique, omitting yet their deep unity. 
It was shown recently [16] that equation for Stokes vector evolution can be derived in a consequent way from the Maxwell equations on the basis of the quantum-mechanical diagonalization procedure, which generalizes QIA approach. Another derivation of SVF equations - directly from QIA equations - was suggested in [17]

This paper intends to derive equations for the Stokes vector evolution in a simplified way, based on QIA equations. We would like to discuss here distinctions between two approaches under discussion and simultaneously to point out their practical equivalence in conditions, when the total phase of the wave field is not significant. Besides, one more approach is outlined [18], which involves complex polarization angle (CPA). Interrelations between CPA and traditional angular parameters of polarization ellipse are presented and the equation for CPA is derived from QIA equations.

The paper is organized as follows. Sect. 2 outlines the basic equations of quasi-isotropic approximation. Sect.3 derives SVF from QIA equations. Sect.4 compares two approaches under discussion. Sect. 5 derives evolution equation for CPA, taking into account both Faraday, and Cotton Mouton effects. Simultaneously Sect.5 points out equivalence between CPA and SVF for narrow beams. At last, stemming from equation for CPA, Sect. 6 derives evolution equations for traditional angular parameters of polarization ellipse.

\section{QUASI-ISOTROPIC APPROXIMATION (QIA)}

Quasi-isotropic approximation (QIA) of the geometrical optics method [3-7] describes propagation of electromagnetic waves in weakly anisotropic media. In similar media all the components of the anisotropy tensor $v_{m n}=\varepsilon_{m n}-\varepsilon_{0} \delta_{m n}$ are small as compared with $\varepsilon_{0}$, where $\varepsilon_{0}$ is an electric permittivity of the isotropic background medium, $\varepsilon_{m n}$ is the full tensor of electrical permittivity and $\delta_{m n}$ is a unit tensor. QIA operates with two small parameters. One of them is traditional "geometrical" small parameter

$$
\mu_{G O}=1 / k_{0} L<<1
$$

where $k_{0}$ is a wave number and $L$ is a characteristic scale of the medium inhomogeneity. Besides, QIA involves anisotropic" small parameter

$$
\mu_{A}=\max \left|v_{m n}\right| / \varepsilon_{0} \ll<,
$$

which characterizes weakness of the medium anisotropy.

According to [3-7], asymptotic solution of the Maxwell equations in the lowest approximation in small parameters $\mu_{G O}$ and $\mu_{A}$ can be presented as

$$
\mathbf{E}=A \boldsymbol{\Gamma} \exp (i k \Psi),
$$

where $A$ and $\Psi$ are correspondingly amplitude and eikonal of the electromagnetic wave in isotropic medium and $\Gamma$ is a polarization vector, which is orthogonal to the reference ray, like in an isotropic medium. Let the unit vectors $\mathbf{e}_{1}$ and $\mathbf{e}_{2}$ together with the tangent unit vector $\mathbf{I}$ form a basis for the Popov's orthogonal coordinate system $\left(\xi_{1}, \xi_{2}, \sigma\right)$, associated with a selected (reference) ray [19] (see also Ch.9 in the book [20] as well as Ch.4 in the book [21]; in the latter one Popov's system appears as "ray centered coordinate system"). Unit vectors $\mathbf{e}_{1}$ and $\mathbf{e}_{2}$ of the Popov's orthogonal system obey the equations

$$
\frac{d \mathbf{e}_{i}}{d \sigma}=-\frac{1}{2}\left(\mathbf{e}_{i} \cdot \nabla \ln \varepsilon_{0}\right) \mathbf{l}, i=1,2 .
$$

The most important feature of these vectors is their ability to describe parallel transport of the electrical vector $\mathbf{E}$ along the reference ray in an isotropic medium.

In the Popov's orthogonal coordinate system the polarization vector $\Gamma$ can be presented as

$$
\boldsymbol{\Gamma}=\Gamma_{1} \mathbf{e}_{1}+\Gamma_{2} \mathbf{e}_{2} .
$$

The components $\Gamma_{1}$ and $\Gamma_{2}$ of polarization vector obey the QIA equations 


$$
\begin{aligned}
& \frac{d \Gamma_{1}}{d \sigma}=\frac{1}{2} i k_{0} \varepsilon_{0}^{-1 / 2}\left(v_{11} \Gamma_{1}+v_{12} \Gamma_{2}\right), \\
& \frac{d \Gamma_{2}}{d \sigma}=\frac{1}{2} i k_{0} \varepsilon_{0}^{-1 / 2}\left(v_{21} \Gamma_{1}+v_{22} \Gamma_{2}\right),
\end{aligned}
$$

where $\sigma$ is an arc length along the reference ray. In distinction to the original form of the QIA equations, written in frame of natural trihedral coordinate system [3-7], equations (6) do not contain torsion of the ray, because the Popov's orthogonal system provides parallel, i.e. torsionless transport of the electrical intensity vector along the ray.

For weakly anisotropic collision plasma permittivity of isotropic background could be written as $\varepsilon_{0}=1-V$ and the components of the anisotropy tensor are given by

$$
\left\{\begin{array}{l}
v_{11}=u V \sin ^{2} \alpha_{\|} \cos ^{2} \alpha_{\perp}+i\left(P-Q \sin ^{2} \alpha_{\|} \cos ^{2} \alpha_{\perp}\right) w \\
v_{12}=(i V \sqrt{u}+R w) \cos \alpha_{\|}+(u V-i Q w) \sin ^{2} \alpha_{\|} \sin \alpha_{\perp} \cos \alpha_{\perp}, \\
v_{21}=-(i V \sqrt{u}+R w) \cos \alpha_{\|}+(u V-i Q w) \sin ^{2} \alpha_{\|} \sin \alpha_{\perp} \cos \alpha_{\perp}, \\
v_{22}=u V \sin ^{2} \alpha_{\|} \sin ^{2} \alpha_{\perp}+i\left(P-Q \sin ^{2} \alpha_{\|} \sin ^{2} \alpha_{\perp}\right) w
\end{array}\right.
$$

where $v=\omega_{p}^{2} / \omega^{2}$ and $u=\omega_{c}^{2} / \omega^{2}$ are standard plasma parameters [2], $\omega_{p}$ and $\omega_{c}$ are plasma frequency and electron cyclotron frequency correspondingly and $P, Q, R, V$ are dimensionless parameters:

$$
P=\frac{v(1+u)}{(1-u)^{2}}, \quad Q=\frac{u v(3-u)}{(1-u)^{2}}, \quad R=\frac{2 v \sqrt{u}}{(1-u)^{2}}, \quad V=\frac{v}{1-u}
$$

Parameter $w$ is a collision frequency $v_{c}$ normalized to sounding microwave frequency $\omega: w=v_{c} / \omega$. Eqs.(7) are linearized in collision parameter $w$, which is considered to be small enough. At last, $\alpha_{\|}$and $\alpha_{\perp}$ are polar and azimuthal angles, characterizing orientation of the magnetic field $\mathbf{B}_{\mathbf{0}}$ in the orthogonal basis $\left(\mathbf{e}_{1}, \mathbf{e}_{2}\right)$, Fig.1.

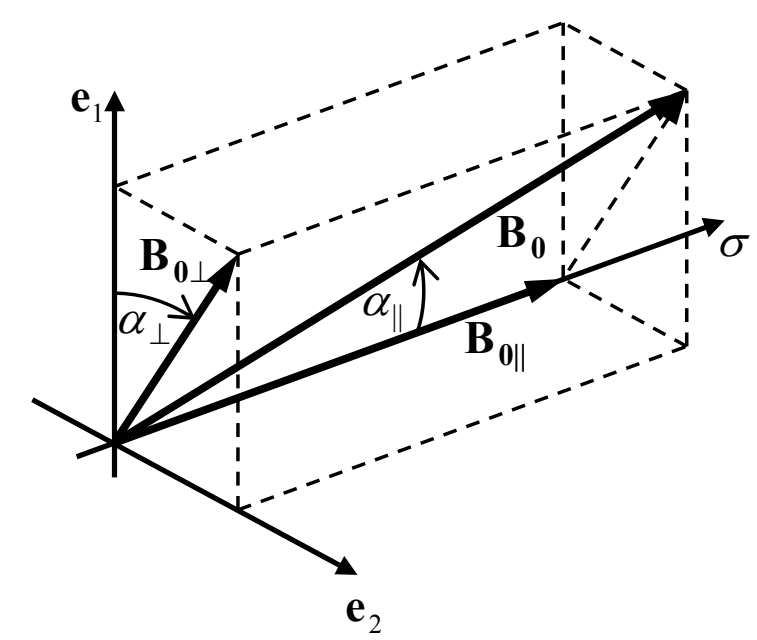

FIGURE 1. Orientation of the static magnetic vector $\mathbf{B}_{0}$ in the orthogonal basis $\mathbf{e}_{1}, \mathbf{e}_{2}$.

\section{EQUATION FOR STOKES VECTOR EVOLUTION DERIVED FROM QIA}

For monochromatic wave field the components of the full (four components) Stokes vector $\mathbf{S}=\left(s_{0}, s_{1}, s_{2}, s_{3}\right)$ are connected with the components of the polarization vector by relations [22]:

$$
s_{0}=\left|\Gamma_{1}\right|^{2}+\left|\Gamma_{2}\right|^{2}, s_{1}=\left|\Gamma_{1}\right|^{2}-\left|\Gamma_{2}\right|^{2}, s_{2}=\Gamma_{1} \Gamma_{2}^{*}+\Gamma_{1}^{*} \Gamma_{2}, s_{3}=i\left(\Gamma_{1}^{*} \Gamma_{2}-\Gamma_{1} \Gamma_{2}^{*}\right)
$$


According to eqs. (9), the derivatives $s_{k}=d s_{k} / d \sigma$ with respect to ark length $\sigma$ are as follows:

$$
\left\{\begin{array}{l}
s_{0}=\dot{\Gamma}_{1} \Gamma_{1}^{*}+\Gamma_{1} \dot{\Gamma}_{1}^{*}+\Gamma_{2} \Gamma_{2}^{*}+\Gamma_{2} \dot{\Gamma}_{2}^{*}, \\
s_{1}=\Gamma_{1} \Gamma_{1}^{*}+\Gamma_{1} \Gamma_{1}^{*}-\Gamma_{2} \Gamma_{2}^{*}-\Gamma_{2} \Gamma_{2}^{*}, \\
s_{2}=\Gamma_{1} \Gamma_{2}^{*}+\Gamma_{1} \dot{\Gamma}_{2}^{*}+\dot{\Gamma}_{1}^{*} \Gamma_{2}+\Gamma_{1}^{*} \Gamma_{2} \\
s_{3}=i\left(\Gamma_{1}^{*} \Gamma_{2}+\Gamma_{1}^{*} \Gamma_{2}-\Gamma_{1} \Gamma_{2}^{*}-\Gamma_{1} \Gamma_{2}^{*}\right) .
\end{array}\right.
$$

For further calculations it is convenient to present the anisotropy tensor as a sum of Hermitian and anti-Hermitian parts:

$$
v_{i j}=v_{i j}^{h}+v_{i j}^{a}, v_{i j}^{h}=\frac{1}{2}\left(v_{i j}+v_{j i}^{*}\right), v_{i j}^{a}=\frac{1}{2}\left(v_{i j}-v_{j i}^{*}\right) .
$$

Using derivatives $\Gamma_{1}, \Gamma_{2}$ from eqs.(6) and taking into account that $v_{i j}^{h} *=v_{j i}^{h}, v_{i j}^{a} *=-v_{j i}^{a}$, one can obtain

$$
\left\{\begin{array}{l}
s_{0}=\frac{k_{0}}{2 \sqrt{\varepsilon_{0}}}\left[i\left(v_{11}^{a}+v_{22}^{a}\right) s_{0}+i\left(v_{11}^{a}-v_{22}^{a}\right) s_{1}+i\left(v_{12}^{a}+v_{21}^{a}\right) s_{2}+\left(v_{12}^{a}-v_{21}^{a}\right) s_{3}\right] \\
s_{1}=\frac{k_{0}}{2 \sqrt{\varepsilon_{0}}}\left[i\left(v_{11}^{a}-v_{22}^{a}\right) s_{0}+i\left(v_{11}^{a}+v_{22}^{a}\right) s_{1}-i\left(v_{21}^{h}-v_{12}^{h}\right) s_{2}+\left(v_{12}^{h}+v_{21}^{h}\right) s_{3}\right] \\
s_{2}=\frac{k_{0}}{2 \sqrt{\varepsilon_{0}}}\left[i\left(v_{12}^{a}+v_{22}^{a}\right) s_{0}+i\left(v_{21}^{h}-v_{12}^{h}\right) s_{1}+i\left(v_{11}^{a}+v_{22}^{a}\right) s_{2}-\left(v_{11}^{h}-v_{22}^{h}\right) s_{3}\right] \\
s_{3}=\frac{k_{0}}{2 \sqrt{\varepsilon_{0}}}\left[\left(v_{12}^{a}-v_{21}^{a}\right) s_{0}-\left(v_{12}^{h}+v_{21}^{h}\right) s_{1}+\left(v_{11}^{h}-v_{22}^{h}\right) s_{2}+i\left(v_{11}^{a}+v_{22}^{a}\right) s_{3}\right]
\end{array}\right.
$$

Following [8-14], let us rewrite eqs. (12) in a compact vector form:

$$
\mathbf{S}=\hat{\mathbf{M S}} \text {, }
$$

where $\hat{\mathbf{M}}$ is a general form of differential Mueller matrix for weakly anisotropic media:

$$
\hat{\mathbf{M}}=\frac{k_{0}}{2 \sqrt{\varepsilon_{0}}}\left\{\begin{array}{cccc}
i\left(v_{11}^{a}+v_{22}^{a}\right) & i\left(v_{11}^{a}-v_{22}^{a}\right) & i\left(v_{12}^{a}+v_{21}^{a}\right) & \left(v_{12}^{a}-v_{21}^{a}\right) \\
i\left(v_{11}^{a}-v_{22}^{a}\right) & i\left(v_{11}^{a}+v_{22}^{a}\right) & -i\left(v_{21}^{h}-v_{12}^{h}\right) & \left(v_{12}^{h}+v_{21}^{h}\right) \\
i\left(v_{12}^{a}+v_{21}^{a}\right) & i\left(v_{21}^{h}-v_{12}^{h}\right) & i\left(v_{11}^{a}+v_{22}^{a}\right) & -\left(v_{11}^{h}-v_{22}^{h}\right) \\
\left(v_{12}^{a}-v_{21}^{a}\right) & -\left(v_{12}^{h}+v_{21}^{h}\right) & \left(v_{11}^{h}-v_{22}^{h}\right) & i\left(v_{11}^{a}+v_{22}^{a}\right)
\end{array}\right\}
$$

This matrix can be presented as a sum of three terms

$$
\hat{\mathbf{M}}=\hat{\mathbf{M}}_{a}+\hat{\mathbf{M}}_{d}+\hat{\mathbf{M}}_{b},
$$

partially remaining representation of Mueller matrix in $[13,15]$. The first, attenuation component,

$$
\hat{\mathbf{M}}_{a}=\frac{k_{0}}{2 \sqrt{\varepsilon_{0}}} i\left(v_{11}^{a}+v_{22}^{a}\left(\begin{array}{cccc}
1 & 0 & 0 & 0 \\
0 & 1 & 0 & 0 \\
0 & 0 & 1 & 0 \\
0 & 0 & 0 & 1
\end{array}\right),\right.
$$

describes attenuation, common for all the component of the Stokes vector The second term 


$$
\hat{\mathbf{M}}_{d}=\frac{k_{0}}{2 \sqrt{\varepsilon_{0}}}\left\{\begin{array}{cccc}
0 & i\left(v_{11}^{a}-v_{22}^{a}\right) & i\left(v_{12}^{a}+v_{21}^{a}\right) & \left(v_{12}^{a}-v_{21}^{a}\right) \\
i\left(v_{11}^{a}-v_{22}^{a}\right) & 0 & 0 & 0 \\
i\left(v_{12}^{a}+v_{21}^{a}\right) & 0 & 0 & 0 \\
\left(\begin{array}{c}
a \\
\left.v_{12}^{a}-v_{21}^{a}\right)
\end{array}\right. & 0 & 0 & 0
\end{array}\right\}
$$

corresponds to the phenomenon of dichroism, that is for selective attenuation of normal modes. At last, matrix

describes birefringence.

$$
\hat{\mathbf{M}}_{b}=\frac{k_{0}}{2 \sqrt{\varepsilon_{0}}}\left\{\begin{array}{cccc}
0 & 0 & 0 & 0 \\
0 & 0 & -i\left(v_{21}^{h}-v_{12}^{h}\right) & \left(v_{12}^{h}+v_{21}^{h}\right) \\
0 & i\left(v_{21}^{h}-v_{12}^{h}\right) & 0 & -\left(v_{11}^{h}-v_{22}^{h}\right) \\
0 & -\left(v_{12}^{h}+v_{21}^{h}\right) & \left(v_{11}^{h}-v_{22}^{h}\right) & 0
\end{array}\right\}
$$

According to eqs.(7), Hermitian and anti-Hermitian parts $\hat{v}^{h}$ and $\hat{v}^{a}$ of the anisotropy tensor $\hat{v}$ are:

$$
\left\{\begin{array}{l}
v_{11}^{h}=u V \sin ^{2} \alpha_{\|} \cos ^{2} \alpha_{\perp} \\
v_{12}^{h}=i V \sqrt{u} \cos \alpha_{\|}+u V \sin ^{2} \alpha_{\|} \sin \alpha_{\perp} \cos \alpha_{\perp} \\
v_{21}^{h}=-i V \sqrt{u} \cos \alpha_{\|}+u V \sin ^{2} \alpha_{\|} \sin \alpha_{\perp} \cos \alpha_{\perp} \\
v_{22}^{h}=u V \sin ^{2} \alpha_{\|} \sin ^{2} \alpha_{\perp}
\end{array},\left\{\begin{array}{l}
v_{11}^{a}=i\left(P-Q \sin ^{2} \alpha_{\|} \cos ^{2} \alpha_{\perp}\right)_{w} \\
v_{12}^{a}=\left(R \cos \alpha_{\|}-i Q \sin ^{2} \alpha_{\|} \sin \alpha_{\perp} \cos \alpha_{\perp}\right)_{w} \\
v_{21}^{a}=\left(-R \cos \alpha_{\|}-i Q \sin ^{2} \alpha_{\|} \sin \alpha_{\perp} \cos \alpha_{\perp}\right)_{w} \\
v_{22}^{a}=i\left(P-Q \sin ^{2} \alpha_{\|} \sin ^{2} \alpha_{\perp}\right)_{w}
\end{array}\right.\right.
$$

Substitution of (19) into general relations (16-18) leads to the representation of differential Mueller matrix for weakly anisotropic magnetized plasma. The term

$$
\hat{\mathbf{M}}_{a}=\frac{k_{0}\left(-2 P+Q \sin ^{2} \alpha_{\|}\right) w}{2 \sqrt{1-V}}\left(\begin{array}{cccc}
1 & 0 & 0 & 0 \\
0 & 1 & 0 & 0 \\
0 & 0 & 1 & 0 \\
0 & 0 & 0 & 1
\end{array}\right)
$$

describes common attenuation for all components of the Stokes vector, the factor $-2 P+Q \sin ^{2} \alpha_{\|}$always being negative. Next term

$$
\hat{\mathbf{M}}_{d}=\frac{k_{0} Q w}{2 \sqrt{1-V}}\left(\begin{array}{cccc}
0 & \sin ^{2} \alpha_{\|} \cos 2 \alpha_{\perp} & \sin ^{2} \alpha_{\|} \sin 2 \alpha_{\perp} & \frac{4}{\sqrt{u(3-u)}} \cos \alpha_{\|} \\
\sin ^{2} \alpha_{\|} \cos 2 \alpha_{\perp} & 0 & 0 & 0 \\
\sin ^{2} \alpha_{\|} \sin 2 \alpha_{\perp} & 0 & 0 & 0 \\
\frac{4}{\sqrt{u(3-u)} \cos \alpha_{\|}} & 0 & 0 & 0
\end{array}\right)
$$

answers to the phenomenon of dichroism. At last, the term

$$
\hat{\mathbf{M}}_{b}=\frac{k_{0} V}{2 \sqrt{1-V}}\left(\begin{array}{cccc}
0 & 0 & 0 & 0 \\
0 & 0 & -2 \sqrt{u} \cos \alpha_{\|} & u \sin ^{2} \alpha_{\|} \sin 2 \alpha_{\perp} \\
0 & 2 \sqrt{u} \cos \alpha_{\|} & 0 & -u \sin ^{2} \alpha \cos 2 \alpha_{\perp} \\
0 & -u \sin ^{2} \alpha_{\|} \sin 2 \alpha_{\perp} & u \sin ^{2} \alpha_{\|} \cos 2 \alpha_{\perp} & 0
\end{array}\right)
$$

corresponds to birefringence. 
Though the expressions (20-22) generally are similar to the Segre's results [13,15], however some explicit distinctions are observed. In particular, the papers $[13,15]$ do not contain the term with Q in Eq. (20) (this term can be substantial when $u$ is not small enough) and the elements of matrix (21) are twice as large as compared with the corresponding matrix elements in $[13,15]$ (the reason is an arithmetic inaccuracy in [13]).

In the case of collisionless plasma, when $w=0$ and $s_{0}=$ const it is convenient to deal with a reduced (three component) Stokes vector $\mathbf{s}=\left(s_{1}, s_{2}, s_{3}\right)[22]$ :

$$
s_{1}=\cos 2 \chi \cos 2 \psi, \quad s_{2}=\cos 2 \chi \sin 2 \psi, \quad s_{3}=\sin 2 \chi .
$$

Here $\psi$ is the azimuth $(0 \leq \psi \leq \pi)$ and $\tan \chi=b / a$ is ellipticity $(-\pi / 4 \leq \chi \leq \pi / 4)$ of polarization ellipse. In this case eq.(13) takes a simplified form

$$
\mathbf{s}=\mathbf{\Omega} \times \mathbf{s},
$$

where $\mathbf{\Omega}=\left(\Omega_{1}, \Omega_{2}, \Omega_{3}\right)$ is a vector with the components [11-14]:

$$
\Omega_{1}=\frac{1}{2} k_{0} V u \sin ^{2} \alpha_{\|} \cos 2 \alpha_{\perp}, \Omega_{2}=\frac{1}{2} k_{0} V u \sin ^{2} \alpha_{\|} \sin 2 \alpha_{\perp}, \Omega_{3}=k_{0} V u^{1 / 2} \cos \alpha_{\|} .
$$

\section{COMPARISON OF QIA EQUATIONS WITH EQUATIONS FOR STOKES VECTOR EVOLUTION}

The equations for Stokes vector, derived from quasi-isotropic approximation of geometrical optics, are very close in form to the equations, obtained in [11-14] on the basis of quite simple if not primitive electrodynamical model, dealing with independent normal modes in locally homogeneous anisotropic medium. In contrast to SVF, QIA stems from Maxwell equations in a consequent way, using an asymptotic expansion of the wave field in small parameters $\mu_{A}$ and $\mu_{G O}$, eqs.(1) and (2).

Equations of QIA determine evolution of polarization vector along the rays, experiencing curvature and torsion, and are able to describe mode conversion due to medium inhomogeneity [3-5]. Therefore equations for the Stokes vector evolution obtained from quasi-isotropic approximation of geometrical optics, acquire all the merits of QIA, in particular, they are able now to describe effects of the ray curvature and torsion in a consequent way, as well as to take into account normal wave interaction and mutual conversion. Another important conclusion is that QIA justifies the equations of SVF, making clear that SVF is valid only for weakly anisotropic plasma.

In spite of high degree of similarity, QIA equations (6) and eqs.(13) for Stokes vector evolution can not be considered as completely identical ones because of phase distinctions. To illustrate this, let us present polarization vector $\boldsymbol{\Gamma}=\left(\Gamma_{1}, \Gamma_{2}\right)=\left(\left|\Gamma_{1}\right| e^{i \delta_{1}},\left|\Gamma_{2}\right| e^{i \delta_{2}}\right)$ as

$$
\Gamma=e^{i\left(\delta_{1}+\delta_{2}\right) / 2}\left(\left|\Gamma_{1}\right| e^{i\left(\delta_{1}-\delta_{2}\right) / 2},\left|\Gamma_{2}\right| e^{i\left(-\delta_{1}+\delta_{2}\right) / 2}\right)
$$

and express four field parameters (two modules $\left|\Gamma_{1}\right|,\left|\Gamma_{2}\right|$ and two phases $\delta_{1}, \delta_{2}$ ) through the components $s_{0}, s_{1}, s_{2}, s_{3}$ of the Stokes vector $\mathbf{S}$. It follows from eqs.(9) that

$$
\left|\Gamma_{1}\right|^{2}=\frac{1}{2}\left(s_{0}+s_{1}\right),\left|\Gamma_{2}\right|^{2}=\frac{1}{2}\left(s_{0}-s_{1}\right), \tan \left(\delta_{1}-\delta_{2}\right)=-\frac{s_{3}}{s_{2}} .
$$

According to eqs.(27), only three field parameters: two modules $\left|\Gamma_{1}\right|,\left|\Gamma_{2}\right|$ and phases difference $\left(\delta_{1}-\delta_{2}\right)$, can be extracted from Stokes vector. It means that four equations (12) for Stokes vector components are not completely equivalent to the QIA equations (6): the phase half sum $\left(\delta_{1}+\delta_{2}\right) / 2$ principally can not be extracted from Stokes vector. The reason is that four components $s_{0}, s_{1}, s_{2}$ and $s_{3}$ of the Stokes vector are connected by a relation $s_{0}^{2}=s_{1}^{2}+s_{2}^{2}+s_{3}^{2}$ and are not independent.

It is worth noticing that in the case of single wave beam the phase $\left(\delta_{1}+\delta_{2}\right) / 2$ does not influence on the shape of polarization ellipse. However, this phase might be important for polarization of the wave field, created by superposition of two or more polarized wave beams. 


\section{COMPLEX POLARIZATION ANGLE (CPA)}

Tangent of CPA is defined as a ratio of complex amplitudes [3-7]: $\tan \gamma=\Gamma_{2} / \Gamma_{1}$. It was shown recently [18], the components $\gamma^{\prime}$ and $\gamma^{\prime \prime}$ of CPA $\gamma=\gamma^{\prime}+i \gamma^{\prime \prime}$ characterize the angular parameters $\psi, \chi$ of the polarization ellipse: real part $\gamma^{\prime}$ defines polarization angle, $\gamma^{\prime}=\psi$ and imaginary part - its ellipticity: $\tanh \gamma^{\prime \prime}=\tan \chi=e$.

It follows from QIA equations (6) that complex polarization angle $\gamma$ in magnetized plasma satisfies the following equation:

$$
\dot{\gamma}=\frac{1}{2} k_{0} V u^{1 / 2} \cos \alpha_{\|}-\frac{1}{4} i k_{0} V u \sin ^{2} \alpha_{\|} \sin \left(2 \gamma-2 \alpha_{\perp}\right) .
$$

Here $\dot{\gamma}=d \gamma / d \sigma$ is a derivative along the ray. This equation can be presented also as [18]:

$$
\dot{\gamma}=\frac{1}{2} \Omega_{3}-\frac{1}{2} i \Omega_{1} \sin 2 \gamma+\frac{1}{2} i \Omega_{2} \cos 2 \gamma=\frac{1}{2} \Omega_{3}-\frac{1}{2} i \Omega_{\perp} \sin \left(2 \gamma-2 \alpha_{\perp}\right),
$$

where $\Omega_{\perp}=\sqrt{\Omega_{1}^{2}+\Omega_{2}^{2}}$. The term $\Omega_{3}$ in eq.(29) corresponds to the Faraday effect, and the term $\Omega_{\perp}$ to Cotton-

Mouton phenomenon. Eqs.(28) and

) for CPA have proved to be very helpful for solution of problems in plasma polarimetry.

\section{EVOLUTION EQUATIONS FOR TRADITIONAL ANGULAR POLARIZATION PARAMETERS}

It follows from Sect. 5 that $\dot{\gamma}^{\prime}=\dot{\psi}, \dot{\gamma}^{\prime \prime}=\dot{\chi} / \cos 2 \chi, \quad \tanh 2 \gamma^{\prime \prime}=\sin 2 \chi, \quad \sinh 2 \gamma^{\prime \prime}=\tan 2 \chi \quad$ and $\cosh 2 \gamma^{\prime \prime}=1 / \cos 2 \chi$. Substituting these relations into equations (29) one can obtain the following system of equations for the traditional angular polarization parameters $(\psi, \chi)$ :

$$
\begin{aligned}
& \dot{\psi}=\frac{\Omega_{3}}{2}+\frac{\Omega_{\perp}}{2} \cos \left(2 \psi-2 \alpha_{\perp}\right) \tan 2 \chi, \\
& \dot{\chi}=-\frac{\Omega_{\perp}}{2} \sin \left(2 \psi-2 \alpha_{\perp}\right) .
\end{aligned}
$$

This system was not known so far. It might be very helpful for plasma polarimetry. Both eq.(30) for CPA and eqs. (33) for angular parameters $\psi$ and $\chi$ are equivalent to the eq (24) for 3-component Stokes vector. In fact, when parameters $\left(\gamma^{\prime}, \gamma^{\prime \prime}\right)$ or $(\psi, \chi)$ being determined from eq. (29) and (30) correspondingly, Stokes vector can be found from eq. (23).

\section{CONCLUSIONS}

Quasi-isotropic approximation of the geometrical optics method provides adequate description for evolution of the polarization vector in inhomogeneous weakly anisotropic plasma. QIA equations take into account: i) curvature and torsion of the ray; ii) longitudinal and lateral inhomogeneity of plasma; iii) torsion of the static magnetic field; iv) weak absorption and dichroism; v) normal modes conversion in the inhomogeneous plasma. It is shown here that QIA equations allow to substantiate Stokes vector formalism and to derive the equation for the Stokes vector evolution in a consequent way. Besides, QIA equations allow deriving the equation for the complex polarization angle as well the equations for angular parameters of polarization ellipse. These equations are shown to be equivalent to the equation for the Stokes vector evolution and promise to become an effective instrument in plasma polarimetry. 


\section{ACKNOWLEDGEMENTS}

This work was supported in part by Association Euratom-IPPLM and Polish Ministry of Science and Higher Education in frame of the project P-12, by STCU in frame of grant P-307 and by CRDF in frame of grant UAM21672-KK-06.

\section{References}

[1] K.G. Budden, Radio Waves in the Ionosphere, Cambridge, Cambridge Univ. Press, 1961.

[2] V.I. Ginzburg, Propagation of Electromagnetic waves in Plasma, New York, Gordon and Breach, 1970.

[3] Yu.A. Kravtsov, Sov. Phys.- Doklady, 13(11), 1125-1127 (1969).

[4] Yu.A. Kravtsov, O.N. Naida and A.A. Fuki, Physics-Uspekhi, 39(2), 129-134 (1996).

[5] A.A. Fuki, Yu.A. Kravtsov and O.N. Naida, Geometrical Optics of Weakly Anisotropic Media, Lond., N.Y., Gordon \& Breach, 1997.

[6] Yu.A. Kravtsov and Yu.I. Orlov, Geometrical optics of inhomogeneous media, Berlin, Heidelberg, Springer Verlag, 1990.

[7] Yu.A. Kravtsov, Geometrical Optics in Engineering Physics, London, Alpha Science, 2005.

[8] G.N. Ramachandran and S. Ramaseshan, Crystal Optics. Encyclopedia of Physics 25/1, Berlin, Springer, 1961.

[9] R.M.A. Azzam, J. Opt. Soc. Am. 68, 1756-1767 (1978).

[10] C.S. Brown and A.E. Bak, Opt. Eng. 34, 1625 (1995).

[11] S.E. Segre, Plasma Phys. Control. Fusion 41, R57-R100 (1999)

[12] S.E. Segre, J. Opt. Soc. Am. A 18, (10), 2601-2606 (2001).

[13] S E Segre, J. Phys. D: Appl. Phys. 36,2806-2810 (2003).

[14] S E Segre and V. Zanza, Plasma Phys. Controlled Fusion 48 339-351 (2006).

[15] S.E. Segre, "Comparison between two alternative approaches for the analysis of polarization evolution of EM waves in a nonuniform, fully anisotropic medium: magnetized plasma," Preprint RT/ERG/FUS/2001/13, 2001, ENEA, Frascati, Italy (2001).

[16] K.Yu. Bliokh, D.Yu. Frolov, and Yu.A. Kravtsov, "Non-Abelian evolution of electromagnetic waves in a weakly anisotropic inhomogeneous medium," Phys. Rev. A, 75, 053821 (2007).

[17] Yu.A. Kravtsov, B. Bieg, K.Yu. Bliokh, J. Opt. Soc. Am. A, 24(10), 3888-3896 (2007).

[18] Z.H. Czyz, B. Bieg and Yu.A. Kravtsov, Phys. Lett. A, 368 101-107 (2007).

[19] M.M. Popov, Vestnik Leningradskogo Universiteta (Bulletin of the Leningrad University), 22, $44-54$ (1969) (in Russian) (Popov's orthogonal coordinate system is described also in [20] and [21]).

[20] V.M. Babich and V.S. Buldyrev, Short-Wavelength Diffraction Theory: Asymptotic Methods, Berlin, Springer Verlag, 1990.

[21] V. Červený, Seismic Ray Theory, Cambridge, Cambridge University Press, 2001.

[22] M.Born and E.Wolf, Principles of Optics ( $7^{\text {th }}$ edition), Cambridge, University Press, 1999. 